\title{
Multiple adverse experiences and child cognitive development
}

\author{
Stephanie A. Guinosso', Sara B. Johnson ${ }^{1,2}$ and Anne W. Riley ${ }^{1}$
}

During childhood and adolescence, children's social environments shape their cognitive development. Children exposed to multiple adversities in their social environment are more likely to have poorer cognitive outcomes. These findings have prompted interest among pediatric and public health communities to screen and connect youth to appropriate interventions that ameliorate the detrimental effects of adverse exposures. Such intervention efforts can be improved with a stronger conceptual understanding of the relationship between multiple adverse exposures and child cognitive development. This includes disentangling adverse exposures from other risk factors or underlying mechanisms, specifying mechanisms of action, and determining when adverse exposures are most detrimental. This review summarizes findings from the literature on each of these areas and proposes a conceptual model to guide further research and intervention.

\section{INTRODUCTION}

Children's social environments shape their cognitive development, including their general cognitive ability and executive functions (i.e., higher-order cognitive abilities including working memory, attention, cognitive flexibility, and planning) $(1,2)$. This development is critical for future health and achievement (3). Given the importance of cognitive development for future success and its malleability in response to environmental input $(1,2)$, there is increasing interest in understanding the mechanisms by which social context and experience influence cognitive outcomes.

Stress or adversity in early life can impair child cognitive performance $(4,5)$. The negative influence of poverty on cognitive outcomes is among the most robust findings in developmental research $(2,6)$. However, other adverse experiences, including abuse, neglect, family instability, parental mental illness, parental substance abuse, parental incarceration, domestic violence, and neighborhood violence, also influence cognitive outcomes (7-9). Data from the National Survey of Children's Health show that nearly one quarter of children younger than $17 \mathrm{y}$ have experienced two or more of these adversities (10). Adversities span social and economic classes, though many are common to conditions of poverty (11).

Children exposed to multiple adverse experiences have worse cognitive outcomes relative to children with any single adverse exposure $(12,13)$. The well-publicized Adverse
Childhood Experiences (ACE) study showed that the number of childhood adverse exposures is associated with health risk behaviors and diseases in adulthood (14). Since the ACE study, evidence has accumulated to support the somewhat intuitive notion that as adversity increases in a young person's life, negative health and developmental outcomes, including cognitive outcomes and achievement, are more likely to occur (9).

These findings have spurred interest among pediatricians and public health practitioners in screening children for early adverse experiences and connecting children to appropriate services $(10,15,16)$. These intervention efforts could be improved with a stronger conceptual model describing the relationship between multiple adverse exposures and child cognitive development. For example, across studies of adverse childhood exposures, the number and types of adversities studied often differ, as do the methodological approaches for examining the relationship between multiple adversities and cognitive outcomes. These differences present challenges for determining those adversities most important for screening and thresholds for referral, as well as underlying mechanisms of action. Mechanisms are critical for informing and focusing intervention efforts.

Timing of adverse exposures may also matter. The brain develops rapidly in childhood, and research from both animal and human models suggests that when an area of the brain is rapidly developing, that region is more sensitive to environmental threats $(4,5,17-19)$. Most studies examining the effects of multiple adverse experiences and developmental outcomes have disregarded the effect of timing of exposure $(9,20)$. Studies are often cross-sectional or assess adverse exposures over a broad developmental period (e.g., before $18 \mathrm{y}$ of age). Thus, there is little empirical evidence about the existence of sensitive periods when children may be particularly vulnerable to multiple adverse exposures $(9,20)$. This is essential for timing prevention programs when they will be most effective.

\section{AIMS AND APPROACH OF CURRENT REVIEW}

This review aims to answer three key questions: First, what are salient adversities to assess in the context of multiple adverse exposures? Second, how do multiple adversities influence cognitive development? Third, what is known about the timing of multiple adverse exposures in relation to cognitive outcomes? The review summarizes findings for each of these questions

\footnotetext{
'Department of Population, Family and Reproductive Health, Johns Hopkins Bloomberg School of Public Health, Baltimore, Maryland; ${ }^{2}$ Department of Pediatrics, Johns Hopkins School of Medicine, Baltimore, Maryland. Correspondence: Stephanie A. Guinosso (switt1@jhu.edu)

Received 5 April 2015; accepted 4 August 2015; advance online publication 11 November 2015. doi:10.1038/pr.2015.195
} 
and proposes a conceptual model to guide future research and interventions in this field. This review is based on a more indepth systematic review (details available upon request) and is limited to studies that assessed the relationship between multiple adverse experiences (defined as at least three different exposures) in relation to either general cognitive ability (such as intellectual functioning or IQ) or specific executive functions (including measures of attention, impulsivity, inhibitory control, and working memory). Adversities were defined as exposures that typically create excessive demands or threats to the child but are preventable or amenable to change, thus lending them to intervention.

\section{SALIENT ADVERSE EXPERIENCES AND COGNITIVE DEVELOPMENT}

The studies in this review assessed various types of adverse exposures. These included maltreatment (abuse or neglect), aspects of family structure and functioning (relationship instability, parental mental health, parental incarceration, parental substance use, housing mobility), poverty-related exposures (crowded dwelling, housing quality and instability, food insufficiency, low income-to-needs), school characteristics (unsafe climate, getting bullied), and neighborhood characteristics (violence exposure). While some studies examined factors across each of these domains, others focused more narrowly. Many studies also examined a number of risk factors (i.e., low birth weight, birth complications, teen or single parent, maternal cognitive ability, or maternal education) and child characteristics (i.e., temperament, behavior problems). This review attempts to disentangle adversities from these other risk factors or child characteristics for the purpose of strengthening our conceptual understanding of how adverse experiences shape cognitive development.

Studies examining the influence of multiple adverse experiences on child cognitive development have generally approached this question in three ways, each of which provides a different perspective on the influence of multiple adverse experiences on cognitive outcomes. Most studies (including the ACE study cited above) use a cumulative index, constructed by dichotomizing each adverse exposure $(1=$ "exposed" and $0=$ "not exposed") and then summing the number of exposures into a single aggregate measure (9). These studies show that cognitive outcomes worsen as the number of adversities increases. This gradient has been observed from infancy through adolescence for outcomes of general cognitive ability (8,21-32). For executive functioning, this gradient has been observed for attention at ages $3-4$ y $(33,34)$, self-regulation at $3 \mathrm{y}$ (35), and delay of gratification at 8-10 y (36). Although the cumulative index is easily understood and communicated to laypersons and policy makers, this approach limits the ability to make inferences about the strength of particular exposures, the relationships between exposures, and the relationship between exposures and mediating factors.

Multiple regression (or ordinary least squares) is a second methodological approach to studying multiple adversities that provides more information about the contribution of individual exposures than does the cumulative index approach. In these models, all adversities are entered into the same regression model, and the significance of each individual adversity, as well as overall variance ( or $R^{2}$ ) explained by the model, is used to evaluate effects. Among studies of general cognitive ability and executive functions, two reported that multiple adverse exposures explained more of the variance in cognitive outcomes than any single adversity $(37,38)$. Additionally, multiple regression models tend to explain more of the variance in cognitive outcomes when compared to a cumulative index approach $(24,28,37)$. Several adversities emerged as significant predictors of child cognitive ability, after accounting for other exposures, including those associated with low socioeconomic status $(22,28,29)$, neighborhood safety (28), and maternal depression (29,39). Among studies of executive function, one study showed that family chaos and instability, but not poverty, was associated with diminished inhibitory control among preschool-aged children (38). Another study looked only at poverty and school characteristics and showed that only poverty was associated with less executive control in children transitioning from preschool to elementary school (40). Yet another study found no effect of six different adversities (including abuse, neglect, parent and school stress, and neighborhood characteristics, etc.) on a range of executive function measures among 10-12 y old children (41). Multiple regression-based studies can be challenging to interpret, especially with small sample sizes, because individual variables that may be otherwise significant will no longer appear so when other, correlated variables are included in the model. Results can overlook important but less influential adversities, leading to seemingly contradictory conclusions (24).

A third, domain-based approach, has also been used to study the effects of multiple adversities on child cognitive development (9). Adversity domains combine adversities of a similar type either by a summative index score or factor analysis. They provide information about dosage of effects as well as insight into the relative salience of particular domains of adversities or the relationship between these domains and mediating factors. For example, Klebanov and Brooks-Gunn examined two maternal psychosocial domains-one human capital (a summative score of maternal employment, education, and welfare status) and the other psychological adversities (a summative score of low social support, maternal depression, and stressful life events) - measured at the time of their child's birth. Although higher scores in both domains separately predicted worse general cognitive ability by simple regression at ages 3 , 5 , and $8 \mathrm{y}$, only the human capital domain predicted cognitive scores at all three ages after accounting for both domains simultaneously (27).

\section{UNDERLYING MECHANISMS}

It is important to distinguish adverse exposures from the underlying mechanisms or processes that may explain how adversities influence developmental outcomes. The quality of the home environment and specific parenting behaviors emerged as important mediators of the relationship between exposure 


\section{Review Guinossoet al.}

to adversity and cognitive outcomes, particularly among children younger than $5 \mathrm{y}$. Among studies of general cognitive ability, children with more socioeconomic adversities were less likely to have stimulating environments (including learning and literacy activities and use of diverse vocabulary) or parents who demonstrated warmth in parenting $(22,25,28,30)$.

McFadden and Tamis-Lemoda explored multiple parenting behaviors under conditions of socioeconomic disadvantage, including poverty, stressful life events, and maternal depression, and found that nonresponsive parenting mediated the effects of maternal depression on the cognitive outcomes of 2 -y-old infants while there was no effect of intrusive or negative parenting (39). Another study showed that the quality of the home environment (i.e., the extent to which the environment is stimulating, safe, and responsive) also mediated the relationship between maternal substance use and cognitive competence (28).

Among mediation studies of executive functions, one showed that maternal warmth and cognitive stimulation in the home both partially mediated the effect of early adversity in the first year of life on attention and behavioral regulation at $3 y$ of age (33). Another study focused on four dimensions of parenting and found that limit-setting and scaffolding (i.e., providing support or direction aligned with the child's needs) mediated the relationship between the cumulative index and executive control among preschoolers, but maternal warmth and negative affect did not (35).

\section{TIMING OF ADVERSE EXPOSURES}

Fewer studies explored the timing of adverse exposures in relation to cognitive development. Five longitudinal studies examined the relationship between adverse exposures measured at a single point in time and concurrent or subsequent general cognitive ability $(26-28,39,42)$. Overall, concurrent adverse exposures were most detrimental to general cognitive ability, and the predictive power diminished over time. However, for the economic hardship domain, the opposite may be true. In one study, poverty-related adversities experienced before $5 \mathrm{y}$ of age had the most pronounced effect on cognitive ability at later time points, most notably when a child entered school (27). Among studies that measured both adversities and cognitive ability at multiple points in time, both adversities and cognitive ability were moderately stable $(8,24)$. One study showed that disparities in cognitive scores between children with high and low levels of adversities increased as children aged, with impairments in cognitive performance evident as early as $14 \mathrm{mo}(22)$.

Four studies examined the relationship between multiple adversities and executive functions over time $(33,35,40,43)$. These suggest that executive functions are malleable and generally improve with age for all youth. However, children with the most adversity earlier in life show the least improvement in executive functions over time and may be more susceptible to school-related adversities that arise in middle school. Only one study explicitly explored the influence of sensitive periods (33). It found that a cumulative index (comprised of economic factors and maternal mental health) at 12 mo explained more of the variance in a composite measure of attention at $3 y$ than did the cumulative index at $3 \mathrm{y}$, providing evidence for the significance of early adversity exposure. Based on these findings, Figure 1 illustrates different developmental trajectories proposed for both cognitive ability and executive function for children with high and low levels of adversity.

\section{A CONCEPTUAL MODEL}

Figure 2 synthesizes these findings into a proposed conceptual model to guide future research and interventions on multiple adversities and cognitive development. With respect to adverse exposures, a domain-based approach is promising because it preserves the simplicity of a cumulative index model by aggregating multiple, highly correlated factors into smaller clusters while also allowing for the examination of relationships across different domains or between specific domains and developmental outcomes (9).

Using a sample of nearly 30,000 adults from the Centers for Disease Control and Prevention's Behavioral Risk Factor Surveillance System, a recent study examined the factor structure (or underlying groupings) of the 11 adversities assessed on the ACE module. The study found evidence for grouping adversities into a household dysfunction domain (consisting of household mental illness, alcohol and substance abuse, incarceration, and parental separation/divorce), a physical and emotional abuse domain, and separate domain for sexual abuse (44). This content-specific grouping provides a useful framework upon which to conceptualize adversity domains. However, the ACE module may also be incomplete in that numerous adversities that may be relevant to child cognitive development are not assessed, including family socioeconomic status.

Building upon this framework and the findings from this review, the proposed conceptual framework describes three domains of adverse exposures. The first pertains to economic hardship (such as living at or below the poverty threshold, living in a crowded dwelling, and having food or housing insecurity). The second domain pertains to family instability. This domain includes adversities such as parental relationship instability, residential mobility, parental mental health or substance abuse disorders, or parental incarceration. The third domain

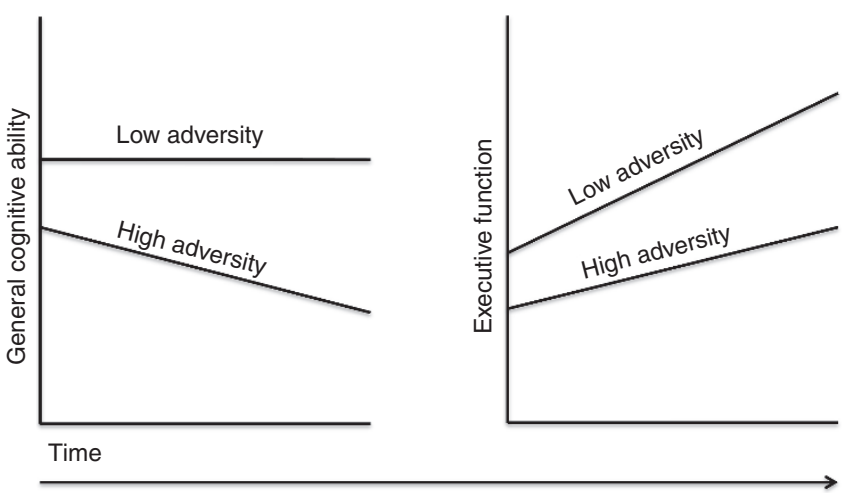

Figure 1. Influence of multiple adversities on general cognitive ability and executive function over time. 


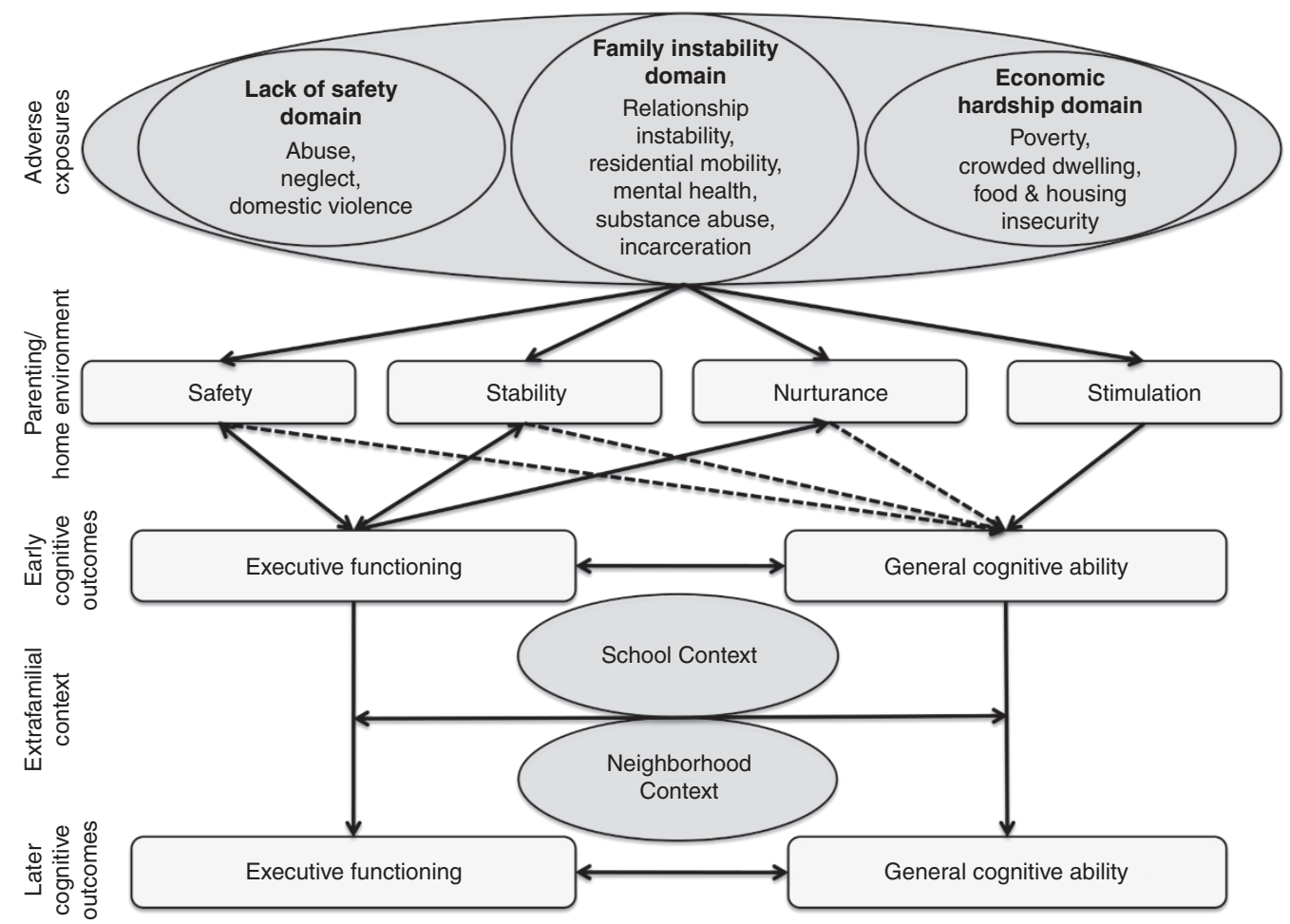

Figure 2. Conceptual model for multiple adverse experiences and cognitive development. Mechanisms not depicted in this model include prenatal exposures (i.e., substance abuse, low birth weight, and early gestational age) $(64,65)$, chemical exposures (such as lead exposure) (66), and malnutrition (6). These exposures are also known to influence cognitive development and may be associated with the adversities described in this model.

includes exposures that directly threaten a child's physical safety, including exposure to abuse, neglect, or domestic violence. While few studies that measured aspects of this third domain met the criteria for this review, there is sufficient research to suggest that exposure to violence or maltreatment has unique detrimental effects on child cognitive development $(5,45,46)$. These domains may have more salience in early childhood, when the child's social context consists primarily of the family. As a child's social world expands, adversities outside of the family context take on a larger role, including schoolrelated adversities (such as bullying or school violence) or neighborhood-level adversities (such as community violence).

These domains influence developmental outcomes through parenting and the home environment in ways that disrupt the safety, stability, nurturance, and stimulation provided to a child. The CDC has developed a framework for the prevention of child maltreatment that emphasizes the importance of safe, stable, and nurturing relationships for healthy development (47). Safety refers to the extent to which a child is free from fear and secure from physical or psychological harm within their environment. Stability refers to the degree of predictability and consistency in a child's environment. Nurturing refers to the extent to which a parent or caregiver is available and able to sensitively and consistently respond to and meet the needs of their child. While not a part of the CDC's model, stimulation is another important mechanism by which adversity can influence child cognitive development. Stimulation refers to the level of learning experiences available in a child's environment.
One of the more consistent findings from this review was the mediating pathway from poverty-related adversities to cognitive ability through cognitive stimulation in the home. A body of research on the effects of poverty on child cognitive development supports this finding. Poverty may limit the capacity of families to invest in stimulating home environments (i.e., books, activities) as well as other resources and services that benefit child health, including nutrition, housing, health care, and child care $(6,48,49)$. In addition, family stress may interfere with parents' ability to allocate time and energy to interact positively with their children, thus influencing the safety, stability, and nurturance provided $(49,50)$. Similarly, unstable home environments, which are often closely linked with poverty, may also impair cognitive development by disrupting positive parent/child interactions (51).

The relations between lack of safe, stable, and nurturing environments and child cognitive development may be partly due to alterations in the stress response. In response to threat, sensory information from the environment is translated into a set of cognitive, behavioral, and physiological responses that are critical to survival; however, continuous engagement of the stress response may inhibit cognitive, behavioral, and physiological adaptation in the long term $(4,52,53)$. One of these responses, the hypothalamic-pituitary-adrenal stress response is well studied (4). Cortisol, the end product of the hypothalamic-pituitary-adrenal cascade, acts throughout the body and brain $(4,45,53)$. Cortisol receptors are densely expressed in the hippocampus, prefrontal cortex, and 
amygdala-regions of the brain that regulate the hypothalamic-pituitary-adrenal axis (4).

Studies have documented atypical stress reactivity among children exposed to multiple adversities $(54,55)$, unsafe environments caused by violence and maltreatment $(5,56,57)$, and poverty (19). Animal and human models suggest that overproduction of cortisol in response to chronic stress and the underproduction of cortisol that may arise from severe deprivation can inhibit neurogenesis in the hippocampus and the prefrontal cortex, negatively impacting learning, memory, and cognition $(4,5,52,53)$. Parent-child interactions can also influence hypothalamic-pituitary-adrenal activity through child attachment. More responsive parenting has been shown to yield more securely attached children, and more securely attached children are less reactive to acute stressors $(52,54,58)$.

\section{TIMING OF INTERVENTIONS}

Developmental research offers differing perspectives with respect to timing of multiple adverse exposures in relation to shaping outcomes later in life (59). The revisionist perspective maintains that early experiences are important in the short term or at the time that the adversities are experienced, but as children mature, they acquire new competencies and are faced with new experiences that weaken the association of early exposures with later outcomes (59). On the other hand, the enduring effects perspective suggests that early experiences are preserved over time, leading to long-term associations of early exposures with later outcomes (59). Enduring effects may be explained by alterations in neurobiological regulatory systems during times of plasticity or by "allostatic load," where chronic or repeated exposure to psychosocial stressors over time leads to wear and tear on the body that results in dysregulation of various regulatory systems, including cognitive functioning (60).

The findings from this review provide some support for both the revisionist and enduring effects perspectives. Among those studies that examined the relationship between adversities at a single point in time and general cognitive ability over time, there was some evidence to suggest that the effects of early adversities diminished, in line with the revisionist perspective. However, among studies that examined both adversities and general cognitive ability over time, there was more support for the enduring effects perspective-i.e., exposure to earlier adversities was associated with diminished cognitive ability or less maturation in executive functions over time. This difference may be attributed to the finding that adversities are likely to persist over time, leading to more continuous or chronic exposures. Only one study from this review explicitly evaluated sensitive periods for cumulative exposures and found evidence for a sensitive period in the development of attention and regulation in the first 14 mo of life (33). This adds to a growing body of evidence that adversity in early childhood has lasting effects on cognitive outcomes (61). However, this does not negate the possible harm of later adverse exposures. Further research is needed to tease apart the differential effects of multiple adversities at a single point in time versus chronic exposure over extended periods of time.

\section{IMPLICATIONS}

The conceptual framework presented here has implications for both research and practice. From a research perspective, the framework proposes relevant domains of adversities for studying cognitive development. The model also distinguishes these domains from other underlying mechanisms. Future research examining these relationships will strengthen our understanding of these pathways and help identify intervention targets. As pediatricians and public health providers embark on increased efforts to screen children for adverse experiences and link them to appropriate care, interventions may be more or less effective, depending on the constellation of adversities to which a young person is exposed. Rather than directing a child to services based on the child's overall adversity score, attention must be given to the types of adversities experienced.

Interventions that promote positive parenting practices in the home environment and enhance cognitive stimulation have been successful. Home visiting programs, for example, are designed to intervene with high-risk families early in a child's life and have improved parenting practices that shape future outcomes for children (62). Additionally, early childhood education programs that aim to provide children with early experiences and stimulation are associated with better cognitive outcomes among children who have experienced adversity $(22,27)$ and have reduced disparities in achievement evident by the time that socioeconomically disadvantaged children enter kindergarten (63). As children age, interventions that promote safe school and neighborhood environments may also buffer the effects of adverse exposures (40).

This review also underscores the need for more research on the timing of adverse exposures in relation to developmental outcomes. While neuroscience indicates that the timing of environmental input can significantly affect developmental pathways $(4,5,18)$, we still know very little about this topic with respect to adverse exposures. The studies included in this review support the notion that both general cognitive ability and executive functioning are shaped by experiences over time, and adverse exposures as early as the first year of life, particularly related to poverty, alter developmental trajectories across childhood. However, more research is needed on the stability and temporal influence of adverse exposures in order to inform the timing of intervention efforts and make the most use of limited intervention resources.

\section{STATEMENT OF FINANCIAL SUPPORT}

Financial support for this study was provided by the Jack Kent Cooke Dissertation Fellowship.

Disclosure: There are no conflicts of interest to disclose.

\section{REFERENCES}

1. Nelson CA, de Hann M, Thomas KM. Neuroscience of Cognitive Development: The Role of Experience and the Developing Brain. Hoboken, NJ: Wiley, 2006.

2. Nisbett RE, Aronson J, Blair C, et al. Intelligence: new findings and theoretical developments. Am Psychol 2012;67:130-59.

3. Noble KG, Tottenham N, Casey BJ. Neuroscience perspectives on disparities in school readiness and cognitive achievement. Future Child 2005;15:71-89. 
4. Lupien SJ, McEwen BS, Gunnar MR, Heim C. Effects of stress throughout the lifespan on the brain, behaviour and cognition. Nat Rev Neurosci 2009;10:434-45.

5. Pechtel P, Pizzagalli DA. Effects of early life stress on cognitive and affective function: an integrated review of human literature. Psychopharmacology (Berl) 2011;214:55-70.

6. Brooks-Gunn J, Duncan GJ. The effects of poverty on children. Future Child 1997;7:55-71.

7. Anda RF, Felitti VJ, Bremner JD, et al. The enduring effects of abuse and related adverse experiences in childhood. A convergence of evidence from neurobiology and epidemiology. Eur Arch Psychiatry Clin Neurosci 2006;256:174-86.

8. Sameroff AJ, Seifer R, Baldwin A, Baldwin C. Stability of intelligence from preschool to adolescence: the influence of social and family risk factors. Child Dev 1993;64:80-97.

9. Evans GW, Li D, Whipple SS. Cumulative risk and child development. Psychol Bull 2013;139:1342-96.

10. Bethell CD, Newacheck P, Hawes E, Halfon N. Adverse childhood experiences: assessing the impact on health and school engagement and the mitigating role of resilience. Health Aff (Millwood) 2014;33:2106-15.

11. Evans GW, Kim P. Multiple risk exposure as a potential explanatory mechanism for the socioeconomic status-health gradient. Ann NY Acad Sci 2010;1186:174-89.

12. Rutter M. Protective factors in children's responses to stress and disadvantage. Ann Acad Med Singapore 1979;8:324-38.

13. Sameroff AJ, Seifer R, Barocas R, Zax M, Greenspan S. Intelligence quotient scores of 4-year-old children: social-environmental risk factors. Pediatrics 1987;79:343-50.

14. Felitti VJ, Anda RF, Nordenberg D, et al. Relationship of childhood abuse and household dysfunction to many of the leading causes of death in adults. The Adverse Childhood Experiences (ACE) Study. Am J Prev Med 1998;14:245-58.

15. Centers for Disease Control and Prevention. Adverse childhood experiences reported by adults---five states, 2009. MMWR Morb Mortal Wkly Rep 2010;59:1609.

16. Anda RF, Butchart A, Felitti VJ, Brown DW. Building a framework for global surveillance of the public health implications of adverse childhood experiences. Am J Prev Med 2010;39:93-8.

17. Shonkoff JP, Boyce WT, McEwen BS. Neuroscience, molecular biology, and the childhood roots of health disparities: building a new framework for health promotion and disease prevention. JAMA 2009;301:2252-9.

18. McEwen BS. Early life influences on life-long patterns of behavior and health. Ment Retard Dev Disabil Res Rev 2003;9:149-54.

19. McEwen BS, Gianaros PJ. Central role of the brain in stress and adaptation: links to socioeconomic status, health, and disease. Ann NY Acad Sci 2010;1186:190-222.

20. Bosch NM, Riese H, Reijneveld SA, et al. Timing matters: long term effects of adversities from prenatal period up to adolescence on adolescents' cortisol stress response. The TRAILS study. Psychoneuroendocrinology 2012;37:1439-47.

21. Alaimo K, Olson CM, Frongillo EA Jr. Food insufficiency and American school-aged children's cognitive, academic, and psychosocial development. Pediatrics 2001;108:44-53.

22. Ayoub C, O'Connor E, Rappolt-Schlictmann G, Vallotton C, Raikes H, Chazan-Cohen R. Cognitive skill performance among young children living in poverty: risk, change, and the promotive effects of Early Head Start. Early Child Res Q 2009;24:289-305.

23. Aro T, Poikkeus AM, Eklund K, et al. Effects of multidomain risk accumulation on cognitive, academic, and behavioural outcomes. J Clin Child Adolesc Psychol 2009;38:883-98.

24. Burchinal MR, Roberts JE, Hooper S, Zeisel SA. Cumulative risk and early cognitive development: a comparison of statistical risk models. Dev Psychol 2000;36:793-807.

25. Burchinal M, Vernon-Feagans L, Cox M; Key Family Life Project Investigators. Cumulative social risk, parenting, and infant development in rural low-income communities. Parent Sci Pract 2008;8:41-69.

26. Hall JE, Sammons P, Sylva K, et al. Measuring the combined risk to young children's cognitive development: an alternative to cumulative indices. $\mathrm{Br} \mathrm{J}$ Dev Psychol 2010;28:219-38.
27. Klebanov P, Brooks-Gunn J. Cumulative, human capital, and psychological risk in the context of early intervention: links with IQ at ages 3, 5, and 8. Ann NY Acad Sci 2006;1094:63-82.

28. Krishnakumar A, Black MM. Longitudinal predictors of competence among African American children: the role of distal and proximal risk factors. J Appl Dev Psychol 2002;23:237-66.

29. Liaw F-R, Brooks-Gunn J. Cumulative familial risks and low-birth weight children's cognitive and behavioral development. J Clin Child Psychol 1994;23:360-272.

30. Poehlmann J. Children's family environments and intellectual outcomes during maternal incarceration. J Marriage Fam 2005;67:1275-85.

31. Biederman J, Milberger S, Faraone SV, et al. Family-environment risk factors for attention-deficit hyperactivity disorder. A test of Rutter's indicators of adversity. Arch Gen Psychiatry 1995;52:464-70.

32. Crozier JC, Barth RP. Cognitive and academic functioning in maltreated children. Child Sch 2005;27:197-206.

33. Mistry RS, Benner AD, Biesanz JC, Clark SL, Howes C. Family and social risk, and parental investments during the early childhood years as predictors of low-income children's school readiness outcomes. Early Child Res Q 2010;25:432-49.

34. Fearon RM, Belsky J. Attachment and attention: protection in relation to gender and cumulative social-contextual adversity. Child Dev 2004;75:1677-93.

35. Lengua LJ, Honorado E, Bush NR. Contextual risk and parenting as predictors of effortful control and social competence in preschool children. J Appl Dev Psychol 2007;28:40-55.

36. Evans GW, English K. The environment of poverty: multiple stressor exposure, psychophysiological stress, and socioemotional adjustment. Child Dev 2002;73:1238-48.

37. Hooper SR, Burchinal MR, Roberts JE, Zeisel S, Neebe EC. Social and family risk factors for infant development at one year: an application of the cumulative risk model. J Appl Dev Psychol 1998;19:85-96.

38. Brown ED, Ackerman BP, Moore CA. Family adversity and inhibitory control for economically disadvantaged children: preschool relations and associations with school readiness. J Fam Psychol 2013;27:443-52.

39. McFadden KE, Tamis-Lemonda CS. Maternal responsiveness, intrusiveness, and negativity during play with infants: contextual associations and infant cognitive status in a low-income sample. Infant Ment Health J 2012;34:80-92.

40. Cybele Raver C, McCoy DC, Lowenstein AE, Pess R. Predicting individual differences in low-income children's executive control from early to middle childhood. Dev Sci 2013;16:394-408.

41. Fishbein D, Warner T, Krebs C, Trevarthen N, Flannery B, Hammond J. Differential relationships between personal and community stressors and children's neurocognitive functioning. Child Maltreat 2009;14:299-315.

42. Laucht M, Esser G, Schmidt MH. Developmental outcome of infants born with biological and psychosocial risks. J Child Psychol Psychiatry 1997;38:843-53.

43. Brown ED, Ackerman BP. Contextual risk, maternal negative emotionality, and the negative emotion dysregulation of preschool children from economically disadvantaged families. Early Educ Dev 2011;22:931-44.

44. Ford DC, Merrick MT, Parks SE, et al. Examination of the factorial structure of adverse childhood experiences and recommendations for three subscale scores. Psychol Violence 2014;4:432-44.

45. Gunnar MR, Barr RG. Stress, early brain development, and behavior. Infants Young Child 1998;11:1-14.

46. De Bellis MD. Developmental traumatology: the psychobiological development of maltreated children and its implications for research, treatment, and policy. Dev Psychopathol 2001;13:539-64.

47. Centers for Disease Control and Prevention. Essentials for childhood: steps to create safe, stable, nurturing relationships and environments. 2014 (http://www.cdc.gov/violenceprevention/pdf/essentials_for_childhood_ framework.pdf).

48. Guo G, Harris KM. The mechanisms mediating the effects of poverty on children's intellectual development. Demography 2000;37:431-47.

49. Yeung WJ, Linver MR, Brooks-Gunn J. How money matters for young children's development: parental investment and family processes. Child Dev 2002;73:1861-79. 
50. Gershoff ET, Aber JL, Raver CC, Lennon MC. Income is not enough: incorporating material hardship into models of income associations with parenting and child development. Child Dev 2007;78:70-95.

51. Schoon I, Jones E, Cheng H, Maughan B. Family hardship, family instability, and cognitive development. J Epidemiol Community Health 2012;66:716-22.

52. Gunnar M, Quevedo K. The neurobiology of stress and development. Annu Rev Psychol 2007;58:145-73.

53. McEwen BS. Central effects of stress hormones in health and disease: understanding the protective and damaging effects of stress and stress mediators. Eur J Pharmacol 2008;583:174-85.

54. Evans GW, Kim P, Ting AH, Tesher HB, Shannis D. Cumulative risk, maternal responsiveness, and allostatic load among young adolescents. Dev Psychol 2007;43:341-51.

55. Lupie SJ, King S, Meaney MJ, McEwen BS. Can poverty get under your skin? basal cortisol levels and cognitive function in children from low and high socioeconomic status. Dev Psychopathol 2001;13:653-76.

56. De Bellis MD. The psychobiology of neglect. Child Maltreat 2005;10: $150-72$.

57. Watts English T, Fortson BL, Gibler N, Hooper SR, De Bellis MD. The psychobiology of maltreatment in childhood. J Soc Issues 2006;62:717-36.

58. Repetti RL, Taylor SE, Seeman TE. Risky families: family social environments and the mental and physical health of offspring. Psychol Bull 2002;128:330-66
59. Roisman GI, Fraley RC. Developmental mechanisms underlying the legacy of childhood experiences. Child Dev Perspect 2013;7:149-54.

60. Taylor SE, Way BM, Seeman TE. Early adversity and adult health outcomes. Dev Psychopathol 2011;23:939-54.

61. Shonkoff JP, Garner AS; Committee on Psychosocial Aspects of Child and Family Health; Committee on Early Childhood, Adoption, and Dependent Care; Section on Developmental and Behavioral Pediatrics. The lifelong effects of early childhood adversity and toxic stress. Pediatrics 2012;129:e232-46.

62. Paulsell D, Avellar S, Martin ES, Del Grosso P. Home visiting evidence of effectiveness review: executive summary. (http://homvee.acf.hhs.gov/ HomVEE_Executive_Summary.pdf) Accessed 15 November 2010.

63. Camilli G, Vargas S, Ryan S, Barnett WS. Meta-analysis of the effects of early education interventions on cognitive and social development. Teach Coll Rec 2010;112:579-620.

64. Huizink AC, Mulder EJ. Maternal smoking, drinking or cannabis use during pregnancy and neurobehavioral and cognitive functioning in human offspring. Neurosci Biobehav Rev 2006;30:24-41.

65. Aarnoudse-Moens CS, Weisglas-Kuperus N, van Goudoever JB, Oosterlaan J. Meta-analysis of neurobehavioral outcomes in very preterm and/or very low birth weight children. Pediatrics 2009;124:717-28.

66. Forns J, Torrent M, Garcia-Esteban R, et al. Longitudinal association between early life socio-environmental factors and attention function at the age 11 years. Environ Res 2012;117:54-9. 